\title{
STRUCTURE OF A SPIN-CROSSOVER Fe(II)-1,2,4-TRIAZOLE POLYMER COMPLEX DISPERSED IN AN ISOTACTIC POLYSTYRENE MATRIX
}

\author{
Miguel Rubio ${ }^{1}$, Rebeca Hernández ${ }^{1}$, Aurora Nogales $^{2}$, Anna Roig $^{3}$, Daniel López ${ }^{1 *}$ \\ ${ }^{1}$ Instituto de Ciencia y Tecnología de Polímeros, CSIC \\ Juan de la Cierva 3, 28006 Madrid, Spain \\ ${ }^{2}$ Instituto de Estructura de la Materia, CSIC \\ Serrano 119, 28006 Madrid, Spain \\ ${ }^{3}$ Institut de Ciència de Materials de Barcelona, CSIC \\ Campus de la UAB, 08193 Bellaterra, Spain
}

\begin{abstract}
*Corresponding author
email:daniel@ictp.csic.es
\end{abstract}

phone: 34915692900

Fax: 34915644853 


\begin{abstract}
Isotactic polystyrene (i-PS) was employed as a matrix to disperse a metallo-organic polymer of $\left.[\mathrm{Fe}(\mathrm{II}) \text { (4-octadecyl-1,2,4-triazole })_{3}\left(\mathrm{ClO}_{4}\right)_{2}\right]$ in order to obtain novel functional materials exhibiting thermal spin crossover transition. A detailed investigation of the structure of the metallo-organic polymer and metallo-organic polymer / iPS blends has been carried out by DSC, WAXD and SAXS techniques as a function of temperature and metallo-organic polymer / iPS proportion.

The results obtained confirm on the one hand that a structural transition associated with a change in the magnetic susceptibilitity of the metallo-organic polymer is preserved in the presence of i-PS. This transition was found to be associated to both, an interconversion of lamellar structures into hexagonal structures and to an increase of intersheet distances within the lamellar structures in metallo-organic polymer films prepared by casting from toluene solutions. On the other hand, an increase of the degree of crystallinity of the iPS is observed in the presence of the metallo-organic polymer which suggests some nucleating effect of the metallo-organic polymer in the crystallization of isotactic polystyrene.
\end{abstract}

\title{
KEYWORDS
}

Metallo-organic polymer; isotactic polystyrene; spin-crossover transition, nucleation 


\section{INTRODUCTION}

One-dimensional polymeric spin-transition (ST) compounds of $\mathrm{d}^{4}-\mathrm{d}^{7}$ transition metal ions are excellent candidates for advanced technological applications such as sensors, displays and information storage [1-4]. These materials can adopt two different magnetic states: a high-spin (HS) and a low-spin (LS) state, which can crossover due to external stimuli such as temperature, pressure, electromagnetic radiation, etc., giving rise to variations in the physical properties of the material like changes in colour, dielectric constant, intramolecular distances or spin state [5-7].

In particular, linear polymeric chains of Fe (II) ions bridged by triazole ligands extensively studied for the past 20 years [8-11] continue to attract considerable attention. This metallo-organic polymer, with Fe (II) ions in an octahedral ligand field, exhibits a thermally induced transition between two electronic states: a diamagnetic $(\mathrm{S}=0)$ LS state and a paramagnetic $(\mathrm{S}=2) \mathrm{HS}$ state. The spin transition is accompanied by a strong thermochromism that provides additional applications for these systems [12$15]$.

The main difficulty for the development of new functional materials is how to transfer the bulk properties of the metallo-organic polymer in the solid state to systems suitable for technological applications. In some cases a micro- or nanopattering of the switchable functional polymers is necessary for the application to be accomplished. In this sense, Langmuir-Blodgett techniques [16-17], layer-by-layer assembly [18], electron beam lithography [1] and self-organization of molecular ST units into hierarchically organized domains across different length scales have been studied [4, 19]. In other cases, the spatial arrangement of the ST polymers is not necessary to attain the desired application and only a dispersion media conferring mechanical and environmental stability [14] to the metallo-organic polymer is required. In this context, 
the ability to isolate and preserve such linear metallo-organic polymers in solution, gels and polymer films constitutes a route to obtain novel functional materials, although it has been little developed so far [20-21].

We recently reported [22] on the gelation properties of [Fe(II) (4-octadecyl-1,2,4triazole $)_{3}\left(\mathrm{ClO}_{4}\right)_{2}$ ] in three organic solvents: toluene, cis- and trans-decalin as a way to obtain easily manipulable and malleable materials for technological applications. The gathering of properties associated to the metallo-organic polymer itself and the general properties of gels (swelling, mechanical, responsive properties) was also sought. The results obtained in this study pointed to structural differences and different gelation mechanisms for the metallo-organic polymer in different solvents and suggested the need to deepen in the structural characterization of the metallo-organic polymer in both, the solid state and the gel state and its dependence with temperature.

The aim of this study is twofold; on the one hand, as a continuation of our previous work, the structure of the Fe (II)-based metallo-organic polymer is analysed as a function of temperature in the solid state. Besides, blends of metallo-organic polymer and isotactic polystyrene were prepared as a route to obtain novel functional materials with improved mechanical properties preserving the bulk properties of the metalloorganic polymer. The resulting systems have been subjected to detailed structural studies by DSC and WAXD and SAXS techniques. 


\section{EXPERIMENTAL PART}

\section{Materials}

The metallo-organic polymer [Fe(II) (4-octadecyl-1,2,4-triazole $\left.)_{3}\left(\mathrm{ClO}_{4}\right)_{2}\right]_{\mathrm{n}}$ was obtained by a method reported previously[22].

Isotactic polystyrene (90\%) was purchased from Scientific Polymer Products, Inc. with a molecular weight of 400,000 g/mol. To obtain amorphous samples, pure iPS was melted at $270{ }^{\circ} \mathrm{C}$ for $10 \mathrm{~min}$ in a hydraulic press and subsequently quenched to room temperature.

The solvent used for the preparation of solutions was toluene supplied by Merck and used without further purification.

\section{Sample Preparation}

Film samples of pure metallo-organic polymer, pure iPS and their blends of different composition were prepared by casting. Homogeneous solutions of both polymers were obtained by mixing the appropriate amounts of polymer and solvent and heating at high temperature until complete dissolution (at $100{ }^{\circ} \mathrm{C}$ for the metallo-organic polymer and at $180{ }^{\circ} \mathrm{C}$ for the iPS). For the blends, homogeneous solutions of each polymer were mixed at $100{ }^{\circ} \mathrm{C}$ and stirred until homogenization. Solutions were poured out into Petri dishes and the solvent was allowed to evaporate in a dessicator at room temperature until constant weight.

Four iPS/ metallo-organic polymer film samples were prepared with different iPS/ complex proportion (w/w): 99.5/0.5, 99/1, 98/2 and 95/5.

\section{Differential scanning calorimetry measurements}

Thermal characterization of the films was conducted through Differential Scanning Calorimetry in a Perkin-Elmer DSC 7 Instrument. Cooling and heating sweeps were performed at a scanning rate of $10^{\circ} \mathrm{C} / \mathrm{min}$. Films were cut into small pieces and put into 
$50 \mu$ aluminium sample pans. Metallo-organic polymer film samples were subjected to the following thermal treatment: heated from $0{ }^{\circ} \mathrm{C}$ to $80{ }^{\circ} \mathrm{C}$, hold at $80{ }^{\circ} \mathrm{C}$ for $1 \mathrm{~min}$ and cooled down to $0{ }^{\circ} \mathrm{C}$ in a subsequent cooling sweep. iPS film samples were subjected to a heating sweep between 0 and $240{ }^{\circ} \mathrm{C}$, hold at $240{ }^{\circ} \mathrm{C}$ for $1 \mathrm{~min}$ and a subsequent cooling down to $0{ }^{\circ} \mathrm{C}$. iPS/complex films have been firstly subjected to a heating sweep between 0 and $80{ }^{\circ} \mathrm{C}$ and quenched to $0{ }^{\circ} \mathrm{C}$, hold for 1 min and performed a second heating sweep to $80{ }^{\circ} \mathrm{C}$ and quenched again to $0{ }^{\circ} \mathrm{C}$, hold for $1 \mathrm{~min}$ and heating in a third sweep to $240{ }^{\circ} \mathrm{C}$.

\section{Magnetic measurements}

The magnetic susceptibility with temperature measurements were carried out using a Quantum Design MPMS5XL SQUID magnetometer operating at 1000 Oe. Measurements were corrected from the diamagnetic contribution of the sample holder. Samples were subjected to cooling and heating cycles between -263 and $60{ }^{\circ} \mathrm{C}$.

\section{Wide angle $X$ ray diffraction}

Wide Angle X ray diffraction (WAXD) experiments were carried out in a Bruker D8 Advance diffractometer equipped with nickel-filtered $\mathrm{CuK}_{\alpha}$ radiation operated at $40 \mathrm{KV}$ and $40 \mathrm{~mA}(\lambda=1.54 \mathrm{~A})$. X-ray diffraction data between 1 and $35^{\circ} 2 \theta$, at room temperature, and between 3 and $35^{\circ} 2 \theta$, for measurements with the temperature sample cell, were collected at scanning rate of $0.2 \mathrm{~s}$ and increment of 0.024 . The profiles were collected using a Gobel mirror equipped with a position-sensitive detector (Vantec 1).

Diffractograms were obtained at different temperatures on heating metallo-organic polymer film samples from $0{ }^{\circ} \mathrm{C}$ to $80{ }^{\circ} \mathrm{C}$. Then, samples were hold at $80{ }^{\circ} \mathrm{C}$ for $1 \mathrm{~min}$ and cooled down to $0{ }^{\circ} \mathrm{C}$ in a subsequent cooling sweep.

iPS/metallo-organic polymer films were subjected to the same thermal protocol followed for DSC experiments: A heating sweep between 0 to $80{ }^{\circ} \mathrm{C}$, obtaining 
diffractograms at different temperatures; quench to $0{ }^{\circ} \mathrm{C}$; hold at $0{ }^{\circ} \mathrm{C}$ for 1 min and a second heating sweep within the same interval of temperature; finally, the sample was quenched again to $0{ }^{\circ} \mathrm{C}$, hold at $0{ }^{\circ} \mathrm{C}$ for 1 min and a third heating sweep was performed between 0 and $240^{\circ} \mathrm{C}$, obtaining spectra at different temperatures.

\section{Small angle $X$ ray scattering}

Small Angle X ray Scattering (SAXS) experiments were carried out on a Bruker AXS Nanostar small-angle $\mathrm{X}$ ray scattering instrument. The instrument uses $\mathrm{CuK} \alpha$ radiation $(1.54 \AA)$ produced in a sealed tube. The scattered $\mathrm{X}$ rays are detected on a two dimensional multiwire area detector and can be converted to one-dimensional scattering by radial averaging and represented as a function of momentum transfer vector $\mathrm{q}$ $(\mathrm{q}=4 \pi \sin \theta / \lambda)$ in which $\theta$ is half the scattering angle and $\lambda$ is the wavelength of the incident X-ray beam.

The raw intensity data $[I(q)]$ were corrected for detector linearity, sample absorption, background scattering, and changes in incident beam intensity. The Bragg long period $\left(L_{\mathrm{B}}\right)$ was determined from the position of the peak in the Lorentz-corrected SAXS intensity, $I(q) q^{2}$. 


\section{RESULTS AND DISCUSSION}

\section{Metallo-organic polymer films}

In the DSC profile shown in Figure 1, the metallo organic complex exhibited an endothermic peak at $\mathrm{T}=51^{\circ} \mathrm{C}$ upon heating. This endothermic peak could be assigned to the spin transition as reported for similar iron (II) complexes [6, 23]. The $\Delta H$ of the endotherm was evaluated to $52 \mathrm{~J} / \mathrm{g}$ in concordance with the enthalpy found for similar systems[23]. Nevertheless, the magnetic characterization of the complex suggests that the main spin-crossover process takes place at lower temperatures.

The magnetic properties of the metallo-organic polymer were measured on cooling and heating over the temperature range of -260 and $60{ }^{\circ} \mathrm{C}$ and the results are depicted in Figure 2. The observation of this figure reveals a low-spin to a high-spin transition occurring in two different steps: a low temperature step, very smooth that occurs without thermal hysteresis at around $-60{ }^{\circ} \mathrm{C}$. This step involves most of Fe (II) ions, provokes a significant change in the magnetic susceptibility and the transition of colour from pink to yellow. The other step takes place more abruptly at around $52{ }^{\circ} \mathrm{C}$, involves only a slight change of the magnetic susceptibility, shows some hysteresis and does not entail any colour change.

The occurrence of two-step SC transitions has been previously reported for different spin crossover systems and has been accounted for by different theories [24-25]. However, these two-step SC transitions usually involve $50 \%$ of Fe (II) ions each. In our case only a small change of the magnetic suspectibility is observed at $52{ }^{\circ} \mathrm{C}$. This suggests that the structural transition at around $52^{\circ} \mathrm{C}$ only provokes a rearrangement of the spatial distribution of spins rather than a spin-crossover transition. Therefore, the genuine spin-crossover transition takes place at lower temperatures, around $-60^{\circ} \mathrm{C}$. 
The WAXD diffractogram corresponding to the metallo-organic polymer recorded at room temperature shown in Figure 3 presents four diffraction peaks at $2 \theta$ values of $2.7^{\circ}$, $5.4^{\circ}, 8.1^{\circ}$ and $21.5^{\circ}$. The first three peaks with Bragg spacings in the ratio 1:2:3 arise from the lamellar structure of the metallo-organic complex. From the first Bragg peak $\left(2 \theta=2.7^{\circ}\right)$ an intersheet spacing of $32 \AA$ can be deduced. Taken into account that the length of the 4-octadecyl-1,2,4-triazole (ODT) is $23 \AA$, the system can be described as layers of transition metal (II) ions intercalated between two layers of ODT. It is important to note that the aliphatic chains in this structure might be interdigitated since the distance between layers of $\mathrm{Fe}(\mathrm{II})(\mathrm{d}=32 \AA)$ is significantly lower than the distance that would correspond to the aliphatic chains without being overlapped (d=46 $\AA$ ) [26]. The broad peak located at $2 \theta=21.5^{\circ}$ that corresponds to a distance $d=4.1 \AA$ has been previously attributed to a two dimensional hexagonal alkyl chain lattice [27-28].

According to these results, a schematic representation (Figure 4a) of the metallo-organic polymer can be proposed in which sheets of rodlike backbones of iron (II) ions are separated by interdigitated alkyl chains. This lamellar organization gives rise to a fibrilar morphology that can be observed by AFM (see supplementary material).

In order to monitor the structural organization of the metallo-organic complex with the temperature, temperature WAXD experiments were carried out. Figure 5a shows the WAXD profiles of the metallo-organic complex upon heating. It is important to remark that for these experiments, the design of the temperature cell is such that it prevents the observation of the first Bragg peak at $2 \theta=2.7^{\circ}$ assigned to the intersheet spacing $(d=32$ A). The behaviour of this peak with temperature will be followed with small angle $\mathrm{X}$ ray scattering as reported in the next section. Taking into account this, the first observable peak in the temperature diffraction patterns is the one located at $2 \theta=5.4^{\circ}$ with the Miller index (200). The thoroughly observation of this peak allows one to infer 
the presence of a shoulder at $2 \theta=6.5^{\circ}$. This shoulder can be accounted for as the second Bragg reflexion of an hexagonal structure with an interlayer spacing of $d=24 \AA$ (considering that for an hexagonal structure the Bragg reflexions peaks fulfil the relation $\left.1:(3)^{1 / 2} \ldots ..\right)$ [29]. See Figure $4 b$ for a schematic representation of the hexagonal structure. Therefore, the metallo-organic polymer films consist of a two-population system of lamellar and hexagonal structures with a higher proportion of lamellar structures.

At temperatures above $50{ }^{\circ} \mathrm{C}$, associated with a change in the magnetic susceptibility of the metallo-organic polymer, the peak at $2 \theta=5.4^{\circ}$ shifts to $2 \theta=4.7^{\circ}$ and the (300) diffraction peak at $2 \theta=8.1^{\circ}$ shifts to $2 \theta=7.2^{\circ}$. The position of the diffraction peak corresponding to the hexagonal structure at $2 \theta=6.5^{\circ}$ does not shift. On the other hand, the intensity of the diffraction peaks associated to the lamellar structure decreases whereas the intensity of the reflections linked to the hexagonal structure increases at temperatures above $50^{\circ} \mathrm{C}$.

According to this, the increase of temperature above $50{ }^{\circ} \mathrm{C}$ provokes both, a change in the lamellar structure of the metallo-organic polymer whose intersheet spacing increases from $32 \AA$ to $40 \AA$ and the transformation of part of the lamellar structures to hexagonal structures (see Figure 4). On the other hand, the position and intensity of the broad peak between $2 \theta=17.5^{\circ}$ and $21.5^{\circ}$ does also vary with temperature: a shoulder can be observed at temperatures below $30{ }^{\circ} \mathrm{C}$. This is in agreement with a two-population system and indicates that the alkyl hexagonal chain packing is also affected. Therefore, the changes in the magnetic susceptibility found at around $52{ }^{\circ} \mathrm{C}$ (see Figure 2) would be associated to the changes of the lamellar structure of the iron (II) chains and not to the breaking up of packing of the alkyl side chains of the triazole ligand. 
The diffraction patterns of the metallo-organic complex taken upon cooling to room temperature (Figure 5b) demonstrates that the structural transition is reversible as denoted by the fact that the two peaks located at $2 \theta=4.7$ and $7.2^{\circ}$ transform into the peak at $2 \theta=5.4^{\circ}(200)$ and the (300) diffraction peak at $2 \theta=8.1^{\circ}$ at temperatures below $\mathrm{T}=35$ ${ }^{\circ} \mathrm{C}$.

Figure 6 shows the SAXS scattering patterns corresponding to the metallo-organic complex upon heating. At temperatures above $50{ }^{\circ} \mathrm{C}$, the peak at $\mathrm{q}=0.2 \AA^{-1}$, characteristic of the distance between layers shifts to $\mathrm{q}=0.16 \AA^{-1}$ indicating that the distance between layers increases in agreement to similar systems based on Fe(II)-1,2,4triazole complexes[14]. Interestingly, the scattering peak at $\mathrm{T}=50{ }^{\circ} \mathrm{C}$ presents a broad shoulder at low q-values whereas the scattering peak at $\mathrm{T}=55^{\circ} \mathrm{C}$ exhibit a shoulder at high q-values. This result might indicate that in this temperature range $\left(\mathrm{T}=50-55{ }^{\circ} \mathrm{C}\right)$ the system is a mixture of low-spacing lamellar structures and high-spacing lamellar structures, plus the corresponding hexagonal structures. It is important to note that the first Bragg reflexion associated to the hexagonal structure (second Bragg reflexion at $6.5^{\circ}$ observed by WAXD) that should appear at $\mathrm{q}=0.26 \mathrm{~A}^{-1}$ cannot be observed as it is out of the experimental limits of the SAXS instrument.

\section{2. iPS/metallo-organic polymer films}

DSC experiments carried out on a 1/99 (g metallo-organic polymer/g iPS) highlight that the presence of iPS does not prevent the structural transition of the metallo-organic complex (Figure 7). However the transition shifts to higher temperatures (at around 60 $\left.{ }^{\circ} \mathrm{C}\right)$ with respect to the transition exhibited by the pristine metallo-organic complex and the associated enthalpy is significantly lower, as expected due to dilution effects. After quenching to $0{ }^{\circ} \mathrm{C}$, the DSC thermogram did not show any transition suggesting that the presence of iPS avoid the structural transition of the complex to be reversible with 
temperature, probably due to a solubilisation of the complex inside the iPS matrix. A third heating scan performed on the same sample quenched to $0{ }^{\circ} \mathrm{C}$ showed a melting endotherm corresponding to the iPS, which demonstrates that the iPS crystallizes in the presence of the metallo-organic complex. Moreover, the peak position $\left(\mathrm{T}_{\mathrm{m}}=223{ }^{\circ} \mathrm{C}\right)$ and the enthalpy associated to the melting of iPS $(\Delta \mathrm{H}=24 \mathrm{~J} / \mathrm{g})$ do not change due to the presence of the metallo-organic polymer, as demonstrated by the comparison of the DSC thermograms of the mixture and the pure iPS, respectively (results not shown here).

The WAXD diffractograms taken at $\mathrm{T}=20^{\circ} \mathrm{C}$ and at $\mathrm{T}=60{ }^{\circ} \mathrm{C}$ for a mixture in proportion 5/95 (g metallo-organic polymer/g iPS) are shown in Figure 8a. As can be observed, the diffraction peak located at $2 \theta=5.4^{\circ}$ in the diffractogram at $\mathrm{T}=20{ }^{\circ} \mathrm{C}$ disappears at $\mathrm{T}=60^{\circ} \mathrm{C}$, hence, this peak can be assigned to the second order Bragg peak (200) of the lamellar morphology of the metallo-organic polymer in the mixture.

Figure $8 \mathrm{~b}$ shows the WAXD diffractograms as a function of temperature upon quenching the $5 / 95$ film to $0{ }^{\circ} \mathrm{C}$. No peak corresponding to the metallo-organic complex is observed in the diffractogram at $\mathrm{T}=20^{\circ} \mathrm{C}$ but six diffraction peaks corresponding to the iPS crystalline structure [30] at $2 \theta=8,14,16,18,22$ and $24^{\circ}$ were identified with Miller indices (110), (300), (220), (211), (411) and (321), respectively. These results are in agreement with the DSC thermograms and they indicate on the one hand that the lamellar morphology of the metallo-organic complex does not reform in the presence of iPS and on the other hand that the iPS crystallinity is preserved. At temperatures above $220{ }^{\circ} \mathrm{C}$, the iPS melting temperature, two broad halos are observed at $2 \theta \approx 18.9^{\circ}$ that arises from phenyl-phenyl correlations and at $2 \theta \approx 9.5^{\circ}$ due to intermolecular correlations of backbone atoms [31]. 
The relative crystallinity $X_{\mathrm{c}, \text { WAXs, }}$, was obtained from the integrated intensity over the observed Bragg reflections (after subtraction of the amorphous background), normalized by the intensity integrated over the full WAXS profile.

Results corresponding to the film compositions (5/95 and 1/99) were compared to the crystallinity of pristine iPS film in Figure 9. At temperatures below the iPS glass transition $\left(\mathrm{Tg}=81^{\circ} \mathrm{C}\right.$, as determined by DSC) the relative crystallinity does not change with temperature for any of the samples and the presence of the metallo-organic complex induces an increase of the relative crystallinity with respect to pristine iPS. At temperatures above the glass transition temperature (around $81{ }^{\circ} \mathrm{C}$ for the pure iPS and around $90{ }^{\circ} \mathrm{C}$ for the $5 / 95$ sample), the relative crystallinity decreases sharply for pure iPS and 5/95 samples. Afterwards, an increase of the relative crystallinity with temperature is obtained for both samples till the melting temperature at around $220{ }^{\circ} \mathrm{C}$. There is no change is the relative crystallinity in the whole temperature range for the sample with a composition $1 / 99$.

The decrease of crystallinity with temperature can be accounted for by the melting of the lowest stability portion of the polystyrene lamella [31]. Then, the melting fraction of polystyrene can recrystallised in a more stable form, giving rise to an increase in the relative crystallinity. Both, the increase in the relative crystallinity in relation to pure iPS and its invariance with temperature for the sample 1/99 suggest the occurrence of a heterogeneous nucleation process induced by the metallo-organic polymer. These results could be interpreted as follows: At low concentration of the metallo-organic polymer, the dilution effect provokes most of the complex being present as individual fibrils that can act as nucleating agents for the crystallisation of iPS. At higher polymer complex concentrations phase separation occurs: 3D crystallites of polymer complex are obtained that cannot nucleate the crystallisation of iPS. As the temperature increases 
above the structural transition of the complex, individual fibrils could be obtained that might induce the crystallisation of iPS.

In order to study in more detail the influence of temperature on the structure of the metallo-organic complex mixed with iPS, SAXS experiments were carried out on films with different metallo-organic polymer/ iPS contents (1/99; 2/98 and 5/95).

For SAXS conducted on iPS at $30{ }^{\circ} \mathrm{C}$ and $80{ }^{\circ} \mathrm{C}$, no discernible SAXS intensity maximum but a monotonously decreasing profile is observed (Figure 10a). The absence of a scattering maximum is attributed on the one hand, to the negligible electron contrast between the lamellar and amorphous layers and on the other hand to the fact that SAXS measurements are carried out at temperatures below $\mathrm{Tg}$.

Figure $10 \mathrm{~b}$ shows the SAXS patterns of the 5/95 (g metallo-organic polymer/g iPS) at $\mathrm{T}=30{ }^{\circ} \mathrm{C}$ and at $\mathrm{T}=80{ }^{\circ} \mathrm{C}$. At $\mathrm{T}=30^{\circ} \mathrm{C}$, the scattering peak at $\mathrm{q}=0.19 \AA$ 年 corresponding to the intersheet spacing of the metallo-organic complex is observed which indicates that its lamellar morphology is maintained upon mixing with iPS. A broad shoulder is located at $\mathrm{q}=0.06 \AA$ which could be attributed to a long period of iPS even if pristine iPS does not present a scattering peak at this temperature (see Figure 10a). This would indicate the formation of iPS crystals with long-range order in the presence of the metallo-organic complex and therefore it suggests a nucleating effect. The intensity I(q) corrected with the Lorentz factor $\mathrm{q}^{2}$ is shown in the inset. The maximum corresponding to the first Bragg reflexion associated to the lamellar structure of the complex shifts from $\mathrm{q}=0.19 \mathrm{~A}^{-1}$ to $\mathrm{q}=0.17 \mathrm{~A}^{-1}$ indicating an increase of the inter-sheet distance from $\mathrm{d}=32 \AA$ to $\mathrm{d}=37 \AA$. Furthermore, the scattering intensity of the first Bragg reflexion at $\mathrm{T}=80{ }^{\circ} \mathrm{C}$ is increased relative to the scattering intensity at $\mathrm{T}=30{ }^{\circ} \mathrm{C}$ indicating that the magnitude of the electron density fluctuation is significantly increased as a consequence of the increase in the crystalline fraction. 
Similar results are obtained for the 2/98 (g metallo-organic polymer/g iPS) film (Figure 10c).

The results obtained are significantly different when the metallo-organic polymer concentration is further decreased as can be observed in figure 10d for the $1 / 99(\mathrm{~g}$ metallo-organic polymer/g iPS). At $\mathrm{T}=30{ }^{\circ} \mathrm{C}$, no peak corresponding to the metalloorganic complex is observed and in addition, a broad peak at $\mathrm{q}=0.09 \mathrm{~A}^{-1}$ is readily visible which could be attributed to the iPS. The fact that this diffraction peak is shifted towards higher q-values and that the scattering intensity greatly increases with respect to the SAXS patterns exhibited by the films with a higher metallo-organic complex shown in Figures 10b and 10c indicates, on the one hand, that the iPS crystalline size is smaller and, on the other hand, an increase of crystallinity with the addition of the metalloorganic complex. Results suggests that the lower the metallo-organic complex concentration in the iPS, the higher the nucleating effect and this is possibly due to the absence of self-organization of the metallo-organic polymer into 3D structures at these concentrations, and supports the explanation given above for the nucleation process to some extent.

\section{CONCLUSIONS}

The structure of films of [Fe(II) (4-octadecyl-1,2,4-triazole $\left.)_{3}\left(\mathrm{ClO}_{4}\right)_{2}\right]_{\mathrm{n}}$ polymer obtained by casting from toluene solutions consists of a two-population mixture of lamellar and hexagonal structures in which lamellar structures are the major component. The heating of the polymer films above $51{ }^{\circ} \mathrm{C}$ entails a thermal transition whose effect is twofold: some lamellar structures transform into hexagonal structures and other lamellar structures undergo an increase of their inter-sheet distances. The transition is thermally 
reversible and the cooling down of the films makes the system to recover the original state.

Metallo-organic polymer / iPS blend films can be obtained by casting from mixtures of solutions of both polymers in toluene. The presence of iPS in the system does not prevent the metallo-organic polymer from crystallising but shifts the thermal transition associated with a change of the magnetic susceptibility to higher temperatures. Nevertheless, the presence of iPS impedes this thermal transition to be reversible as in the case of pristine metallo-organic polymer film.

The effect of the metallo-organic polymer in the crystallisation properties of iPS is more conspicuous: the degree of crystallinity of the iPS increases due to the presence of the metallo-organic polymer. The effect is more pronounced at low metallo-organic polymer concentrations. SAXS experiments also reveal the formation of iPS crystals of smaller size and with long-rage order in the presence of the metallo-organic polymer. Both results suggest a nucleating effect of the metallo-organic polymer in the crystallisation of iPS.

\section{ACKNOWLEDGMENTS}

Financial support from CICYT (MAT2008-01073 and MAT2008-03232) and Fundación Domingo Martínez is gratefully acknowledged. M.R. thanks the Ministerio de Ciencia e Innovación (Spain) for his FPI fellowship. R.H thanks CSIC for a JAE postdoctoral contract. Authors acknowledge E. Verde and M. Hernández for WAXD experiments and AFM images, respectively. 


\section{REFERENCES}

[1] Bousseksou A, Molnar G, Demont P, Menegotto J. Observation of a thermal hysteresis loop in the dielectric constant of spin crossover complexes: towards molecular memory devices. J Mater Chem. 2003;13(9):2069-2071.

[2] Cavallini M, Bergenti I, Milita S, Ruani G, Salitros I, Qu ZR, et al. Micro- and Nanopatterning of Spin-Transition Compounds into Logical Structures. Angew Chem Int Ed. 2008;47(45):8596-8600.

[3] Kahn O, Martinez CJ. Spin-Transition Polymers: From Molecular Materials Toward Memory Devices. Science. 1998;279(5347):44-48.

[4] Ruben M, Rojo J, Romero-Salguero FJ, Uppadine LH, Lehn JM. Grid-type metal ion architectures: Functional metallosupramolecular arrays. Angew Chem Int Ed. 2004;43(28):3644-3662.

[5] Beattie JK. Dynamics of Spin Equilibria in Metal-Complexes. Adv Inorg Chem. 1988;32:1-53.

[6] Fujigaya T, Jiang DL, Aida T. Switching of Spin States Triggered by a Phase Transition: Spin-Crossover Properties of Self-Assembled Iron(II) Complexes with Alkyl-Tethered Triazole Ligands. J Am Chem Soc. 2003;125(48):14690-14691. [7] Spiering H, Kohlhaas T, Romstedt N, Hauser A, Bruns-Yilmaz C, Kusz J, et al. Correlations of the distribution of spin states in spin crossover compounds. Coord Chem Rev. 1999;192:629-647.

[8] Gutlich P. Spin Crossover in Iron(Ii)-Complexes. Structure and Bonding. 1981;44:83-195.

[9] Konig E, Ritter G, Kulshreshtha SK. The Nature of Spin-State Transitions in Solid Complexes of Fe(II) and the Interpretation of Some Associated Phenomena. Chem Rev. 1985;85(3):219-234.

[10] Mikami M, Konno M, Saito Y. The structure changes and the spin phase transition mechanism of a spin crossover complex, [Fe(2-pic)3]Cl2 $E$ tOH. Chem Phys Lett. 1979;63(3):566-569.

[11] Philippe G, Létard J-F, Yufit DS, Chasseau D, Bravic G, Goeta AE, et al. Structural approach of the features of the spin crossover transition in iron(II ) compounds. J Mater Chem. 1999;9:985-994.

[12] Bodenthin Y, Schwarz G, Tomkowicz Z, Geue T, Haase W, Pietsch U, et al. Liquid Crystalline Phase Transition Induces Spin Crossover in a Polyelectrolyte Amphiphile Complex. J Am Chem Soc. 2009;131(8):2934-2941.

[13] Kahn O, Krober J, Jay C. Spin Transition Molecular Materials for Displays and Data Recording. Adv Mater. 1992;4(11):718-728.

[14] Keita K, Tomoko S, Sono S, Masaaki O, Atsushi T, Toyoki K, et al.

Supramolecular control of spin-crossover phenomena in lipophilic Fe(II)-1,2,4-triazole complexes. J Polym Sci A Polym Chem. 2006;44(17):5192-5202.

[15] Kume S, Kuroiwa K, Kimizuka N. Photoresponsive molecular wires of FeII triazole complexes in organic media and light-induced morphological transformations. Chem Comm. 2006(23):2442-2444.

[16] Armand F, Badoux C, Bonville P, Ruaudel-Teixier A, Kahn O. Langmuir-Blodgett Films of Spin Transition Iron(II) Metalloorganic Polymers. 1. Iron(II) Complexes of Octadecyl-1,2,4-triazole. Langmuir. 2002;11(9):3467-3472.

[17] Soyer H, Mingotaud C, Boillot ML, Delhaes P. Spin crossover of a LangmuirBlodgett film based on an amphiphilic iron(II) complex. Langmuir. 1998;14(20):58905895 . 
[18] Cobo S, Molnar G, Real JA, Bousseksou A. Multilayer sequential assembly of thin films that display room-temperature spin crossover with hysteresis. Angew Chem Int Ed. 2006;45(35):5786-5789.

[19] Coronado E, Galán-Mascarós JR, Monrabal-Capilla M, García-Martínez J, PardoIbáñez P. Bistable Spin-Crossover Nanoparticles Showing Magnetic Thermal Hysteresis near Room Temperature. Adv Mater. 2007;19(10):1359-1361.

[20] Lee SW, Lee JW, Jeong SH, Park IW, Kim YM, Jin JI. Processable magnetic plastics composites--spin crossover of PMMA/Fe(II)-complexes composites. Synthetic Metals. 2004;142(1-3):243-249.

[21] Jeong SH. Storing spin-crossover and LC phase transitions information by hybridizing spin-crossover complexes with a thermotropic polymer matrix - A novel case of multiple switching. Molecular crystals and liquid crystals. 2007;471(1):3-10. [22] Rubio M, López D. Effect of solvent on the gelation properties of a metalloorganic polymer of [Fe(II) (4-octadecyl-1,2,4-triazole)3(ClO4)2]n. Eur Polym J. 2009;45(12):3339-3346.

[23] Fujigaya T, Jiang D-L, Aida T. Spin-Crossover Dendrimers: Generation NumberDependent Cooperativity for Thermal Spin Transition. J Am Chem Soc.

2005;127(15):5484-5489.

[24] Garcia Y, Kahn O, Rabardel L, Chansou B, Salmon L, Tuchagues JP. Two-Step Spin Conversion for the Three-Dimensional Compound Tris(4,4'-bis-1,2,4triazole)iron(II) Diperchlorate. Inorg Chem. 1999;38(21):4663-4670.

[25] Molnár G, Guillon T, Moussa NO, Rechignat L, Kitazawa T, Nardone M, et al. Two-step spin-crossover phenomenon under high pressure in the coordination polymer Fe(3-methylpyridine)2[Ni(CN)4]. Chem Phys Lett. 2006;423(1-3):152-156.

[26] Roubeau O, Agricole B, Clerac R, Ravaine S. Triazole-Based Magnetic Langmuir\&\#x2212;Blodgett Films:\&nbsp; Paramagnetic to Spin-Crossover Behavior. J Phys Chem B. 2004;108(39):15110-15116.

[27] Kurth DG, Meister A, Thunemann AF, Forster G. Structure of a Liquid Crystalline Metallosupramolecular Polyelectrolyteâ^'Amphiphile Complex at the Nanoscopic Level. Langmuir. 2003;19(10):4055-4057.

[28] Thunemann AF, General S. Poly(ethylene imine) n-Alkyl Carboxylate Complexes. Langmuir. 2000;16(24):9634-9638.

[29] Hamley IW, Castelletto V. Small-angle scattering of block copolymers in the melt, solution and crystal states. Prog Polym Sci. 2004;29:909-948.

[30] Baskaran N, Hui X, Ince BS, Peggy C. Molecular relaxation of isotactic polystyrene: Real-time dielectric spectroscopy and X-ray scattering studies. J Polym Sci B Polym Phys. 2004;42(5):777-789.

[31] Xu H, Cebe P. Transitions from solid to liquid in isotactic polystyrene studied by thermal analysis and X-ray scattering. Polymer. 2005;46(20):8734-8744. 


\section{FIGURE CAPTIONS}

FIGURE 1.- DSC curves on heating at $10{ }^{\circ} \mathrm{C} / \mathrm{min}$ for the metallo-organic polymer.

FIGURE 2.- $\chi_{\mathrm{T}} \cdot \mathrm{T}$ vs $\mathrm{T}$ on cooling and heating modes for the metallo-organic polymer. The inset emphasises the thermal hysteresis of the transition at high temperatures: $(\bigcirc)$ $1^{\text {st }}$ cooling; $(\square) 1^{\text {st }}$ heating and $(\diamond) 2^{\text {nd }}$ cooling. Pictures show the colour change of the polymer with temperature.

FIGURE 3.- WAXD profile of the metallo-organic polymer at room temperature.

FIGURE 4.- Schematic illustration of the supramolecular units structure in the film of the metallo-organic complex.

FIGURE 5.- WAXD profiles of the metallo-organic polymer obtained on heating (a) and on cooling (b) at different temperatures.

FIGURE 6.- SAXS patterns of the metallo-organic polymer obtained on heating at different temperatures. For an easier visualization, the q-region 0.12-0.22 is shown in the inset.

FIGURE 7.- DSC curves obtained on heating at $10^{\circ} \mathrm{C} / \mathrm{min}$ for a film of the mixture metallo-organic polymer / iPS in proportion 1/99 (w/w).

FIGURE 8.- WAXD profiles of a $5 / 95(\mathrm{w} / \mathrm{w})$ film obtained on heating at different Fiitemperatures: (a) First heating sweep at 30 and $60{ }^{\circ} \mathrm{C}$ and (b) Third heating sweep at various temperatures as indicated in the figure.

FIGURE 9.- Crystallinity as a function of temperature obtained from WAXD experiments for (ם) iPS; (•) 5/95 metallo-organic polymer / iPS mixture (w/w) and (A) 1/99 metallo-organic polymer / iPS mixture (w/w).

FIGURE 10.- SAXS profiles of (a) iPS film; (b) 5/95 metallo-organic polymer / iPS mixture (w/w); (c) 2/98 metallo-organic polymer / iPS mixture (w/w) and (d) 1/99 
metallo-organic polymer / iPS mixture (w/w), obtained at $(\bullet) 30{ }^{\circ} \mathrm{C}$ and $(\bigcirc) 80{ }^{\circ} \mathrm{C}$. Lorentz representation in the insets. 


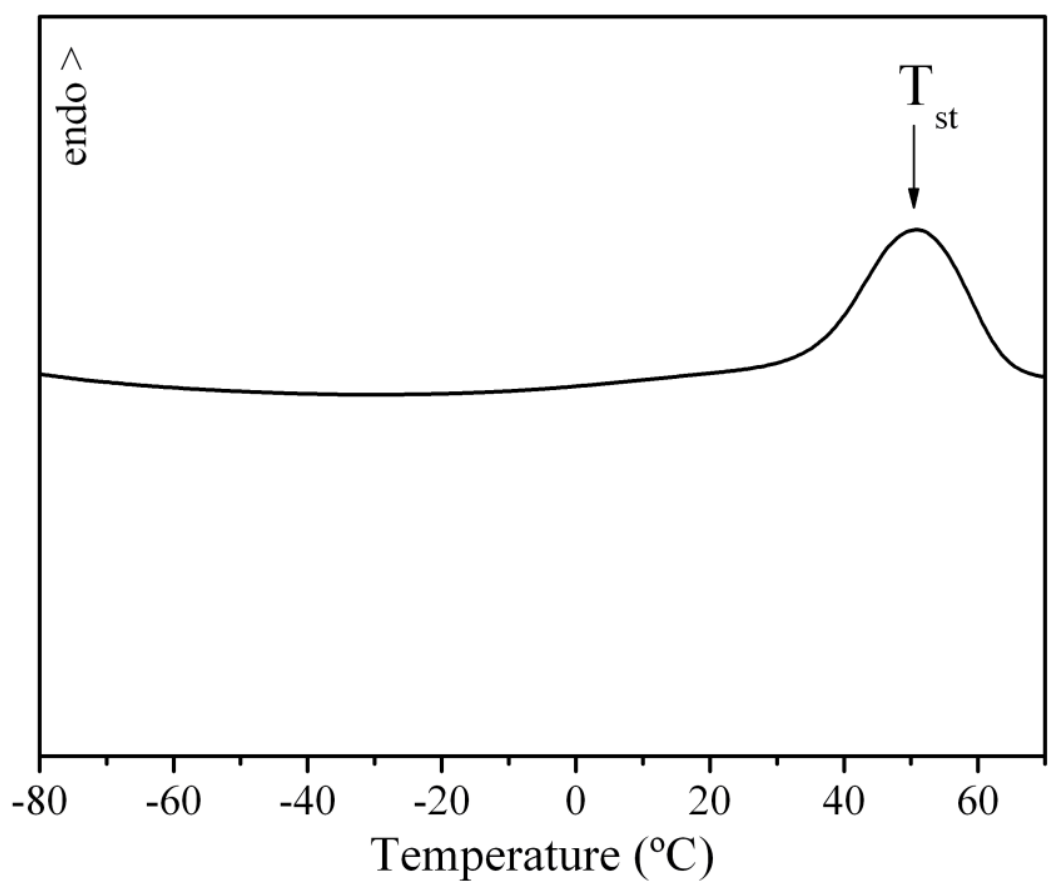

Figure 1 


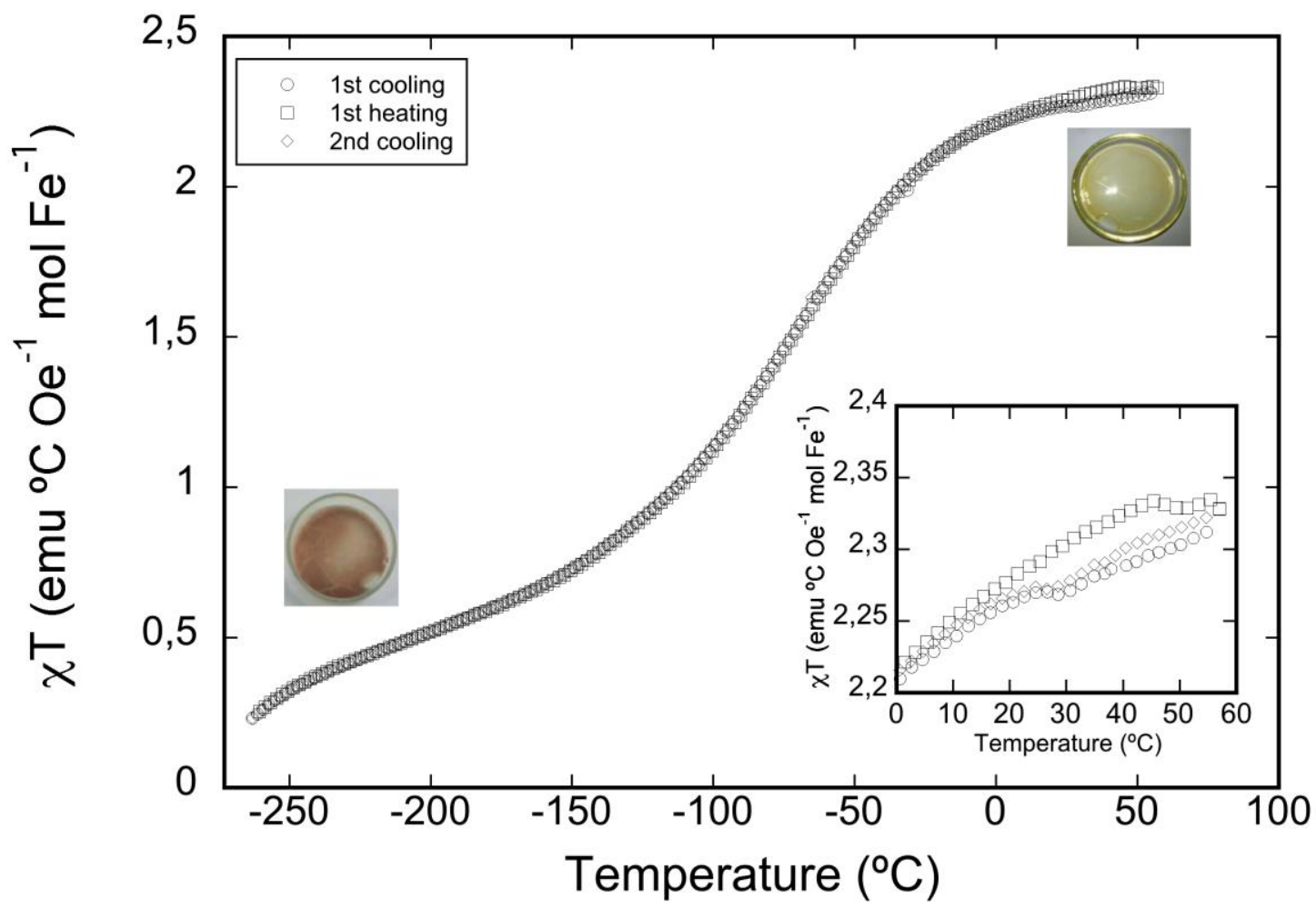

Figure 2 


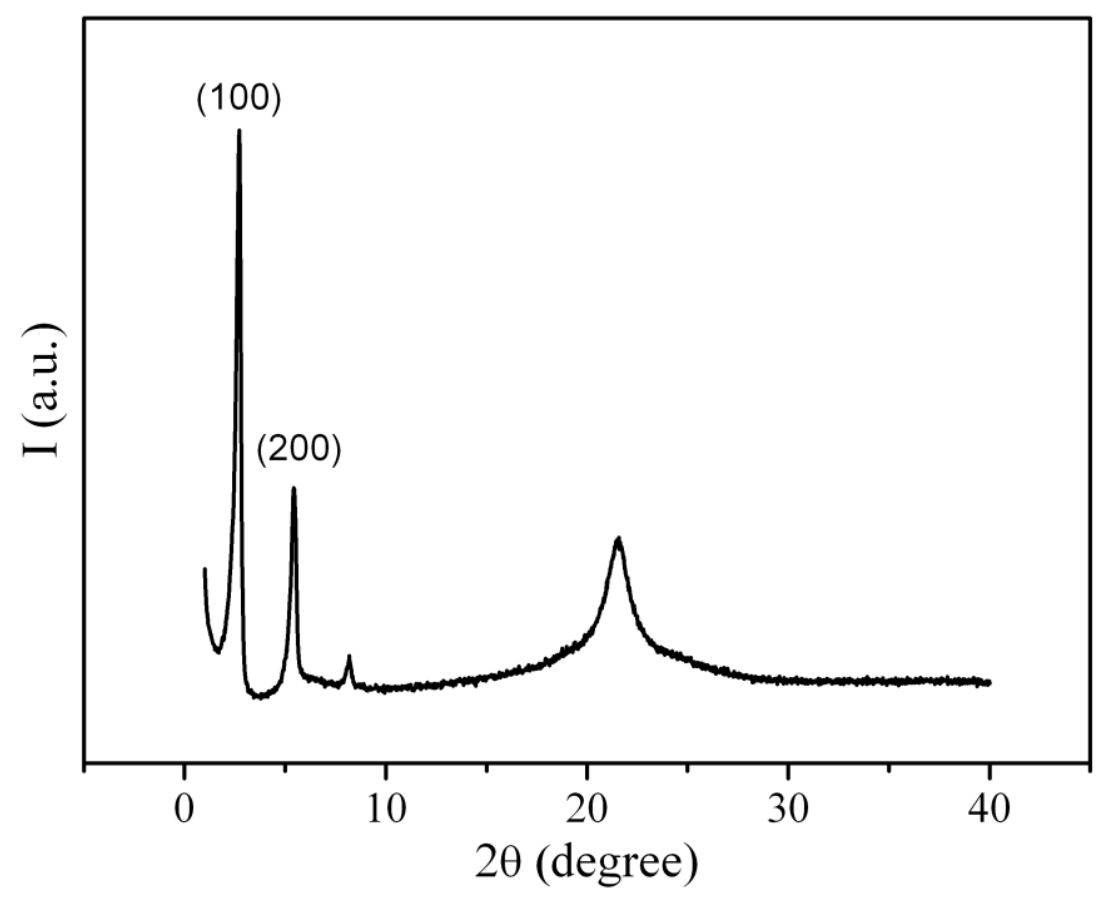

Figure 3 


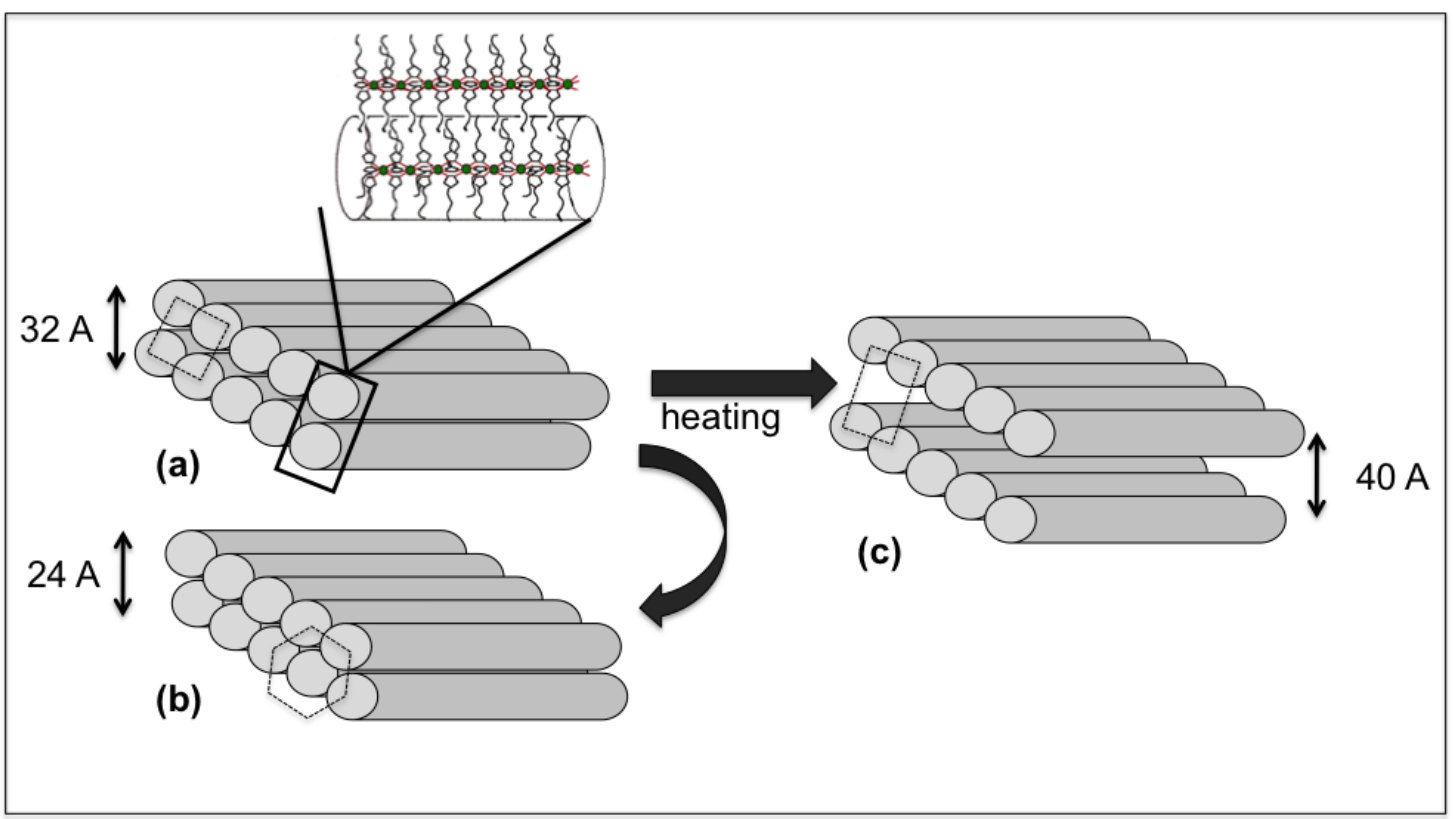

Figure 4 

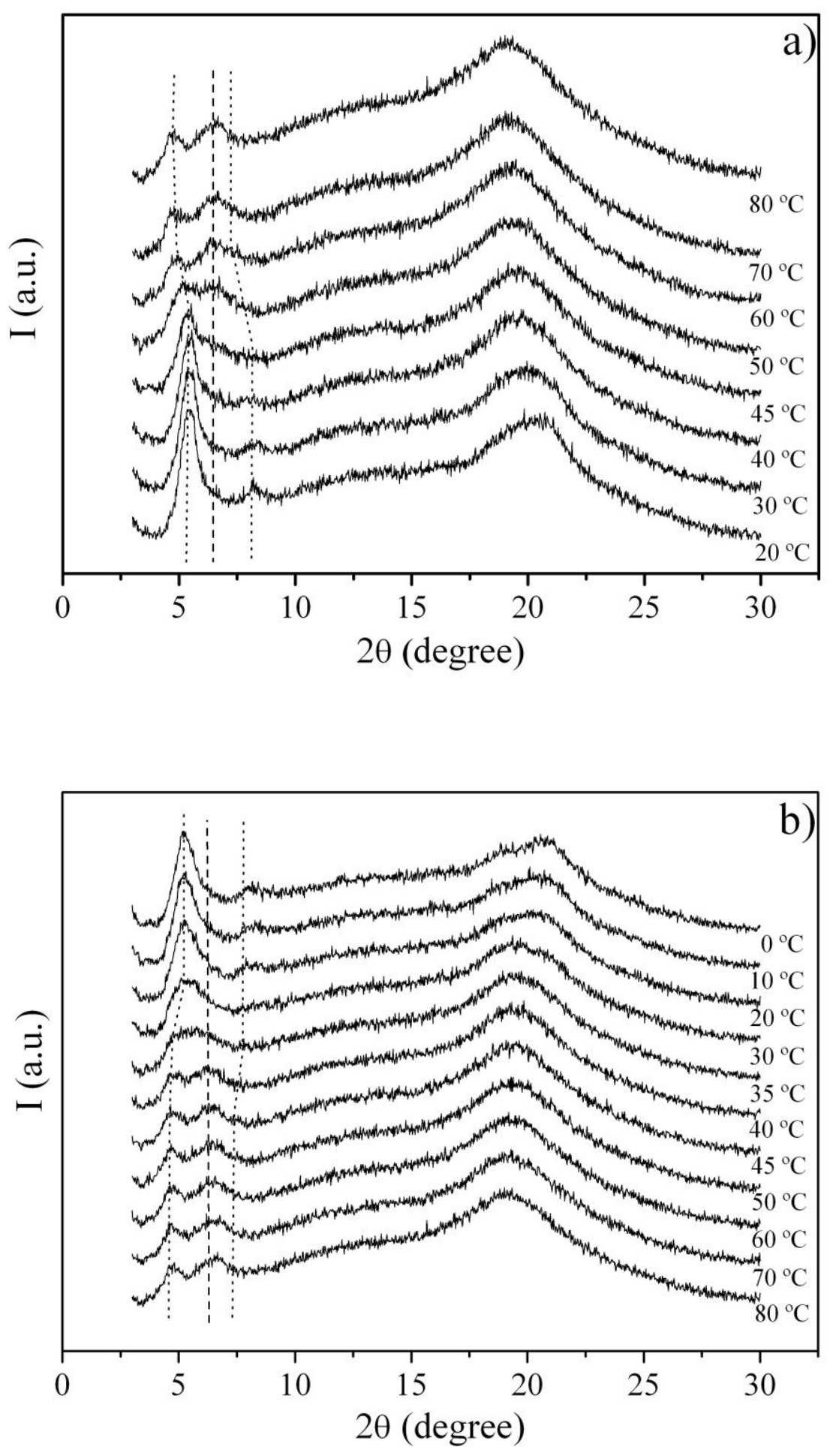

Figure 5 


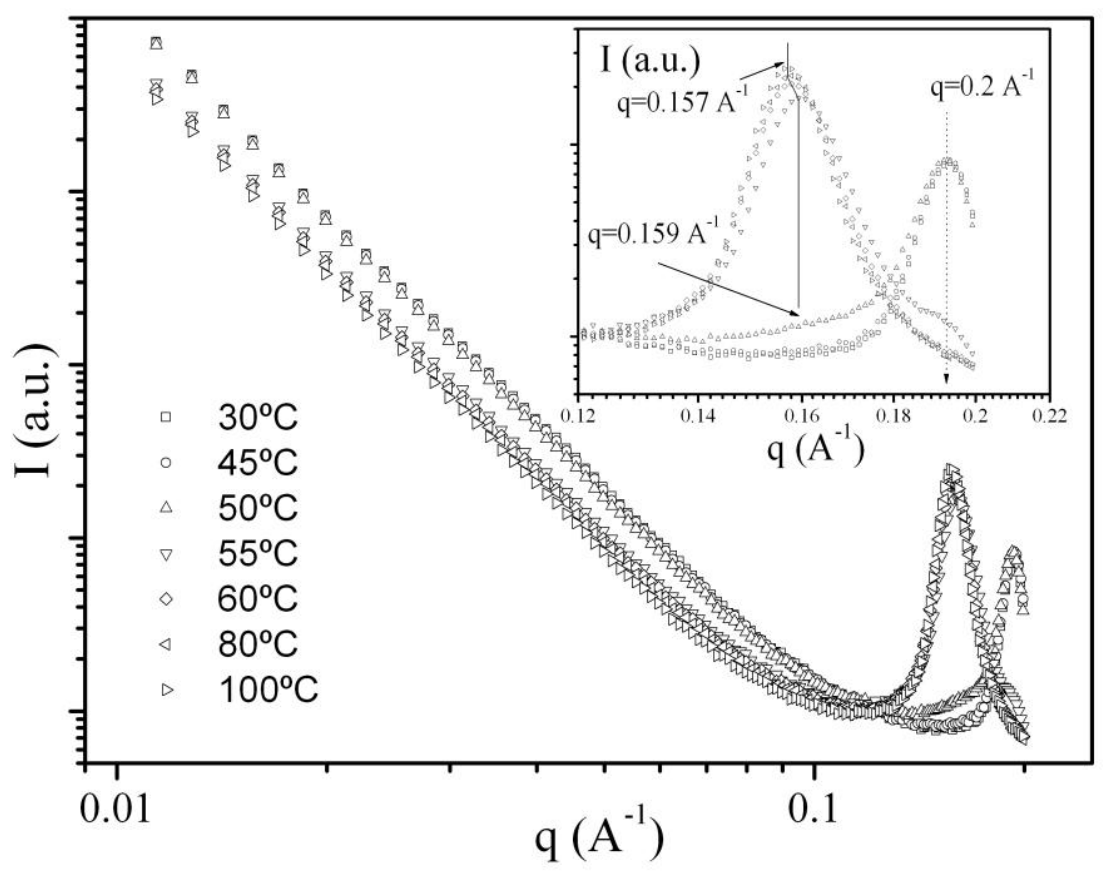

Figure 6 


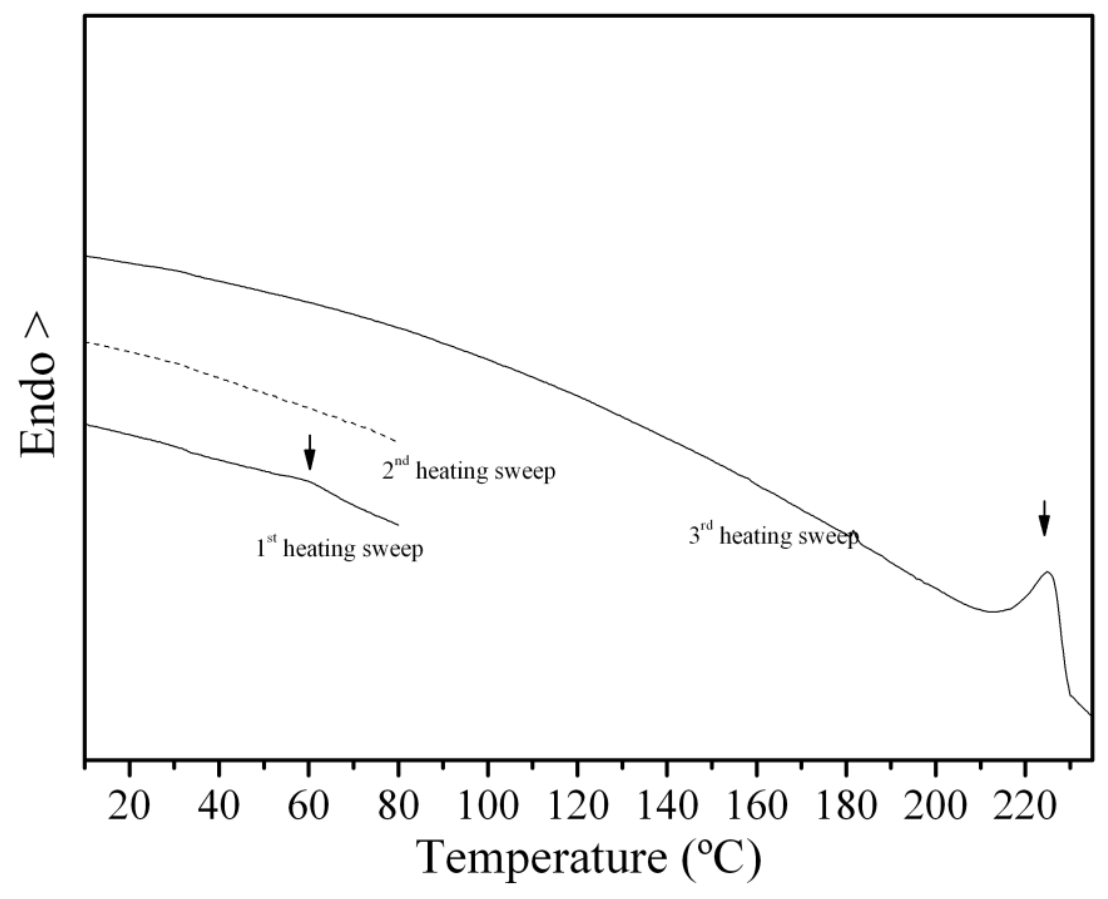

Figure 7 

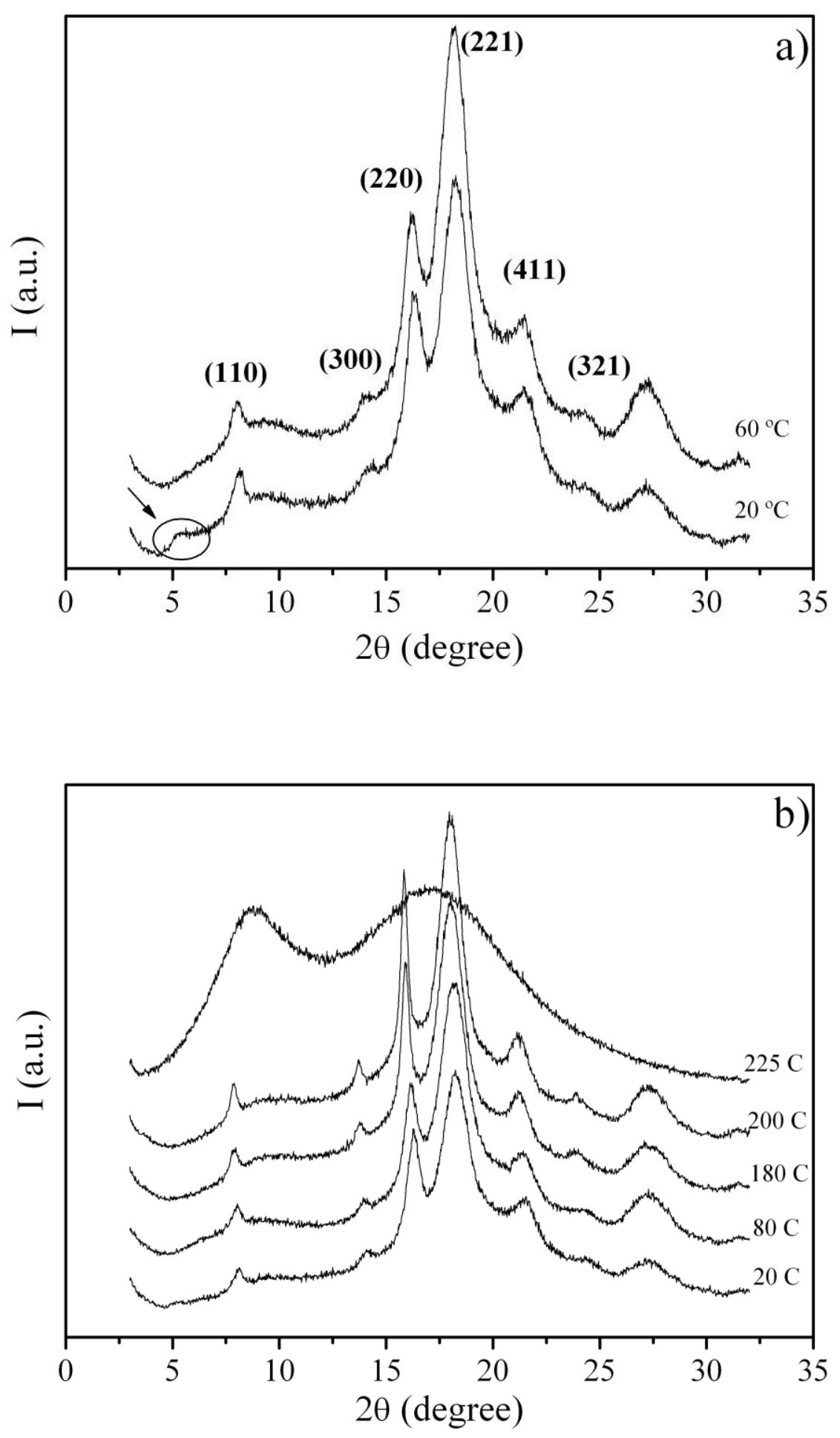

Figure 8 


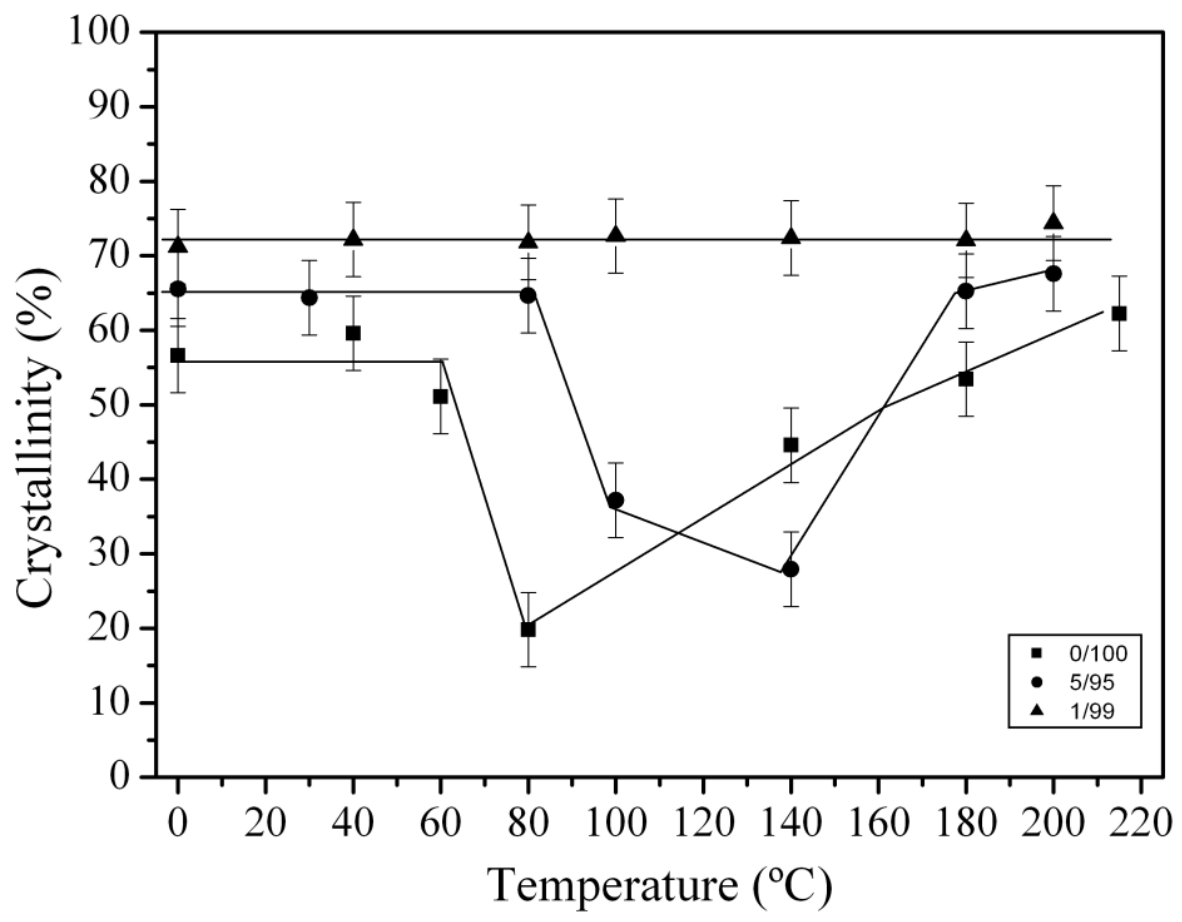

Figure 9 

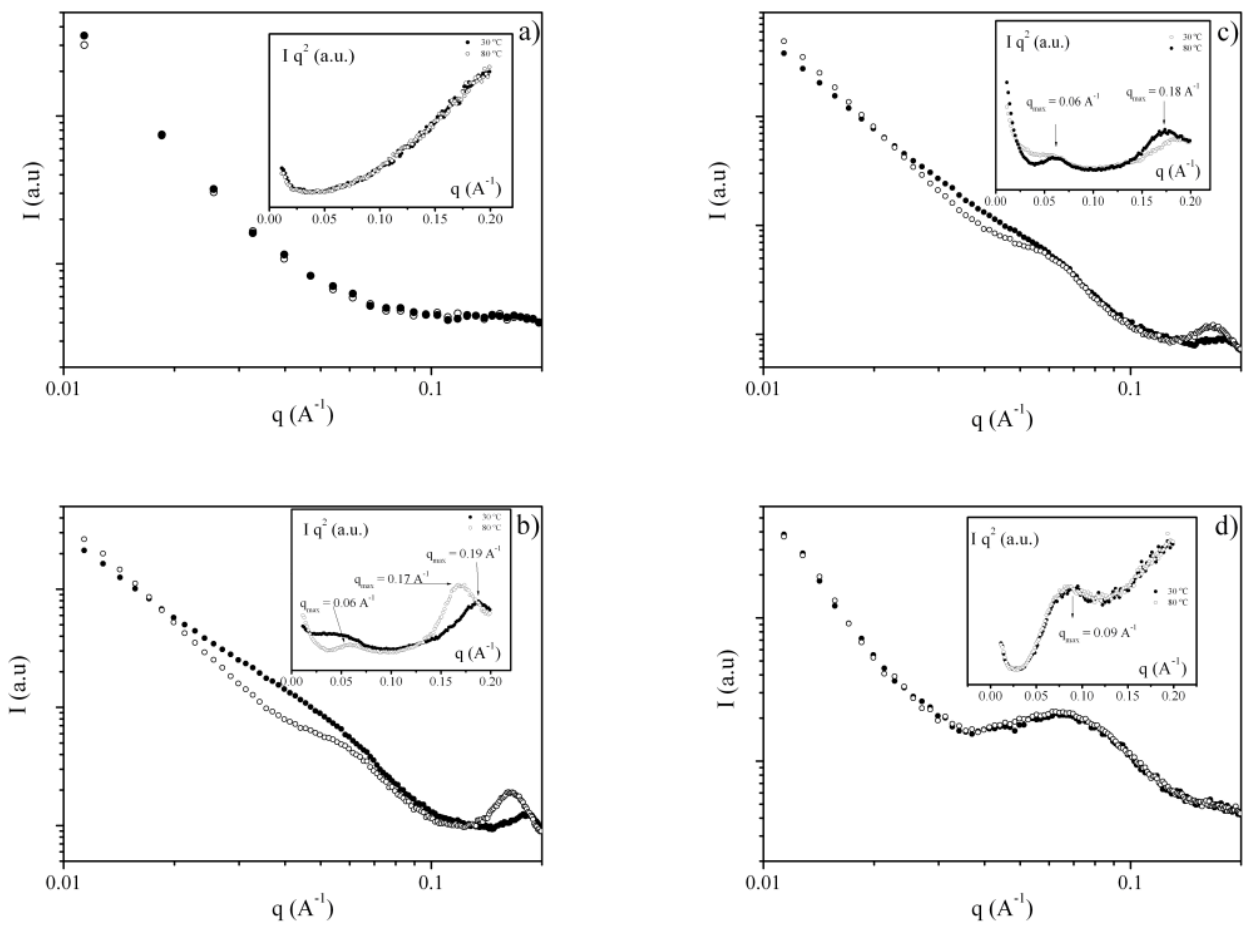

Figure 10 